\title{
Birth defects after assisted reproductive technology according to the method of treatment in Japan: nationwide data between 2004 and 2012
}

\author{
Syuichi Ooki ${ }^{1}$
}

Received: 30 June 2015/ Accepted: 3 August 2015/Published online: 22 August 2015

(C) The Japanese Society for Hygiene 2015

\begin{abstract}
Objectives The purpose of the present study was to analyze birth defects (congenital anomalies) after assisted reproductive technology (ART) according to the method of treatment, namely in vitro fertilization (IVF), intracytoplasmic sperm injection (ICSI) and frozen-thawed embryo transfer (FET).

Methods Individual lists of all ART pregnancies resulting in birth defects from birth year 2004 to 2012 presented in the annual reports by the Japan Society of Obstetrics and Gynecology were used as the initial sources of information. Relative risks (RRs) with the corresponding $95 \%$ confidence intervals (CIs) were calculated with IVF as the reference group when calculating RR of ICSI for IVF, and with FET as the reference group when calculating the RR of fresh embryo transfer for FET.

Results In total, 2725 stillbirths or live births with birth defects were analyzed. The prevalence of birth defects was slightly yet significantly higher in ICSI compared with IVF throughout the study period $(\mathrm{RR}=1.15,95 \% \mathrm{CI}$ $1.02-1.29)$ and in the 2004-2006 period ( $R R=1.26,95 \%$ CI 1.00-1.58). The prevalence of birth defects was significantly higher for fresh embryo transfer compared with FET in the 2004-2006 period $(\mathrm{RR}=1.39$, $95 \% \mathrm{CI}$ 1.12-1.72). The prevalence of birth defects in multiple births was significantly lower in fresh embryo transfer compared with FET $(\mathrm{RR}=0.70,95 \%$ CI $0.55-0.90$, live births of 2007-2012).
\end{abstract}

Syuichi Ooki

sooki@ishikawa-nu.ac.jp

1 Department of Health Science, Ishikawa Prefectural Nursing University, 1-1 Gakuendai, Kahoku, Ishikawa 929-1210, Japan
Conclusions The present descriptive epidemiological study suggests that the impacts of different ART methods on birth defects might differ.

Keywords Birth defects - In vitro fertilization (IVF) . Intracytoplasmic sperm injection (ICSI) - Frozen-thawed embryo transfer (FET) · Nationwide multi-year data

\section{Introduction}

According to Japanese vital statistics, the percentage of live births attributable to assisted reproductive techniques (ART) has increased rapidly, from $0.22 \%$ in 1992 and $1.32 \%$ in 2002 to $3.66 \%$ in 2012. Thus, the use of ART is becoming widespread in Japan.

According to Mayor [1], the risk of congenital malformation in children born after ART is higher than previously thought, and has become a public health issue. There are many epidemiological studies on the relationship between birth defects (also known as 'congenital anomalies' or 'congenital disorders' according to the definition of fact sheets presented by the World Health Organization [2]) and ART. Data from meta-analyses consistently suggest that the overall risk of major birth defects in children born after ART is about $30 \%$ higher than in children conceived spontaneously $[3,4]$. A more recent systematic review and metaanalysis, which analyzed 45 cohort studies, similarly showed that ART infants, including both singletons and multiple births (i.e., twins and triplets/+), had a $32 \%$ higher risk of birth defects compared with naturally conceived infants [5]. Thus, the risk of ART in total on birth defects compared with natural conception is highly probable.

Most of these studies use data from countries where large population-based or hospital-based registries are 
available, for example Scandinavian counties or Australia. On the other hand, data collection of ART, birth defects and births records (vital statistics) are not systematically managed in Japan, and record linkage is virtually impossible [6]. With this background, the author performed secondary data analyses of published information in Japan. The purpose of the present study was to analyze ARTassociated birth defects according to the method of treatment, namely in vitro fertilization (IVF), intracytoplasmic sperm injection (ICSI) and frozen-thawed embryo transfer (FET).

\section{Materials and methods}

\section{Outline of ART-associated birth defect data in Japan}

The method for collecting data has been described elsewhere [6]. Almost all medical institutions performing ART are registered with the Japan Society of Obstetrics and Gynecology (JSOG). The JSOG administers questionnaire surveys for these medical institutions. Some of the survey data such as number of treatment cycles, transfers, pregnancies, deliveries, stillbirths, and live births according to the types of treatment are presented in simple annual reports of aggregate, not individual, data (in Japanese). Fresh embryo transfer was reported according to the two major techniques, IVF and ICSI. FET was reported only as total numbers, including both IVF and ICSI. These annual reports do not include the data on simple ovulation stimulation/enhancement. From birth year 2004 to 2012 (the latest), lists of all ART pregnancies resulting in birth defects have been presented in these annual reports. The presented data include method of treatment (IVF, ICSI and FET), maternal age, perinatal outcome (spontaneous/artificial abortion $(<22$ weeks), stillbirths ( $\geq 22$ weeks), and live births) and gestational week, plurality (singleton, twins, triplets/+, and unknown), sex (male, female, unknown), early neonatal infant death up to day 6 (yes, no, unknown), and names of birth defects and so on. The response rate for ART surveillance between 2004-2012 was $97.7-99.5 \%$, and the mean response rate throughout the 9 years was $99.1 \%$ (5400/5449), meaning that an almost-complete database reflecting the current situation of ART and birth defects in Japan could be constructed.

The author used these case report data as initial information for the present study. The names of birth defects provided in the above annual JSOG lists were carefully checked and reclassified according to the International Classification of Diseases, tenth edition (i.e., ICD-10, 2003 version). Diseases that were classified in the category of ICD-10 code Q00-Q99 (i.e., congenital malformations, deformations and chromosomal abnormalities) were identified as birth defects, and selected and analyzed in the present study. Other congenital diseases not classified in Q00-Q99 were excluded.

\section{Statistical analyses}

This study was designed to compare the risk of birth defects associated with the various micromanipulations of different ART methods, since data on natural conception were not available. First, basic information and perinatal outcome data according to the treatment were presented. Then, the crude prevalence of birth defects calculated as percentage after each ART method per births (stillbirths and live births) were calculated according to three birth year periods (2004-2006, 2007-2009 and 2010-2012). And relative risks (RRs) with the corresponding $95 \%$ confidence intervals (CIs) were calculated with IVF as a reference group when calculating the RR of ICSI for IVF, and with FET as a reference group when calculating RR of fresh embryo transfer for FET.

Singletons and multiple births were combined in the above analysis, since the number of stillbirths according to plurality was not reported in the JSOG data. RRs were calculated according to plurality for live births from 2007 to 2012; this was the only available data on the plurality of total ART.

Statistical analysis was conducted using SAS for Windows ver 9.3 .

\section{Results}

Demographic and perinatal outcome data of ART pregnancies according to the method of treatment are summarized in Table 1. In the process of reclassification of birth defects, 273 out of 3468 (7.9\%) were excluded, since these cases were not classified as ICD-10 code Q00-Q99. Moreover, 470 birth defect cases were excluded because the pregnancy outcome was abortion. In total, 2725 stillbirths or live births with birth defects were included.

Crude prevalence of birth defects and RR with $95 \%$ CI according to the method of treatment (ICSI vs. IVF and fresh embryo transfer vs. FET) by birth year period are shown in Table 2. The prevalence of birth defects was slightly yet significantly higher in ICSI compared with IVF $(\mathrm{RR}=1.15,95 \%$ CI 1.02-1.29) in the total period. Most of this significance disappeared when the time-periods were divided into three classes; only ICSI in the 2004-2006 period showed a significantly higher prevalence $(\mathrm{RR}=1.26,95 \% \mathrm{CI} 1.00-1.58)$. The prevalence of birth defects was significantly higher for fresh embryo transfer 
Table 1 Basic obstetric information according to each treatment

\begin{tabular}{lrrr}
\hline & \multicolumn{2}{c}{ Fresh embryo transfer } & \multicolumn{1}{l}{ FET } \\
\cline { 2 - 3 } & \multicolumn{1}{c}{ IVF } & \multicolumn{1}{c}{ ICSI } \\
\hline Total treatment cycles & 526,567 & 673,148 & 584,731 \\
Total number of transfers & 258,660 & 313,161 & 551,417 \\
Number of pregnancies & 66,748 & 69,069 & 182,701 \\
Number of abortions & 15,451 & 17,215 & 46,163 \\
Number of multiple pregnancies & 6792 & 6203 & 10,978 \\
Number of deliveries & 43,657 & 44,106 & 120,127 \\
Stillbirths & 302 & 306 & 624 \\
Live births & 48,468 & 48,365 & 127,273 \\
Birth defects & & & \\
Abortion & 112 & 100 & 258 \\
Stillbirths & 25 & 31 & 33 \\
Live births & 509 & 579 & 1548 \\
\hline
\end{tabular}

$I V F$ in vitro fertilization, ICSI intracytoplasmic sperm injection, FET frozen-thawed embryo transfer

compared with FET in the 2004-2006 period $(\mathrm{RR}=1.39$, $95 \%$ CI 1.12-1.72).

Crude prevalence of birth defects and RR with $95 \%$ CI according to plurality and the method of treatment by birth year period are shown in Table 3. The prevalence of birth defects in multiple births was significantly lower in fresh embryo transfer compared with FET throughout the study period $(\mathrm{RR}=0.70,95 \% \mathrm{CI} 0.55-0.90)$ and in the 2010-2012 period $(\mathrm{RR}=0.60,95 \%$ CI $0.40-0.91)$.

\section{Discussion}

The present author analyzed birth defects as a short-term outcome indicator of ART, since this was the only available (presented/published) data reflecting the ART outcome for all of Japan. As far as the author knows, this study is the first descriptive epidemiological study in Japan to analyze the relationship between each ART method and birth defects using nationwide multi-year data. The total number of ART-associated deliveries or births was over 200,000 during 9 years of observation.

The present percentage of total birth defects after ART, irrespective of method, may be lower overall compared with other studies mentioned in the reviews [3, 7]. Moreover, the percentage of birth defects after ART is clearly higher in the recent period (2010-2012) than in the earlier period (2004-2006). This is likely not because the frequency of birth defects themselves has rapidly increased, but because reporting bias may have existed (underreporting in the earlier period). Nevertheless, the main objective of this study was to evaluate the prevalence of birth defect according to the ART method, and not to

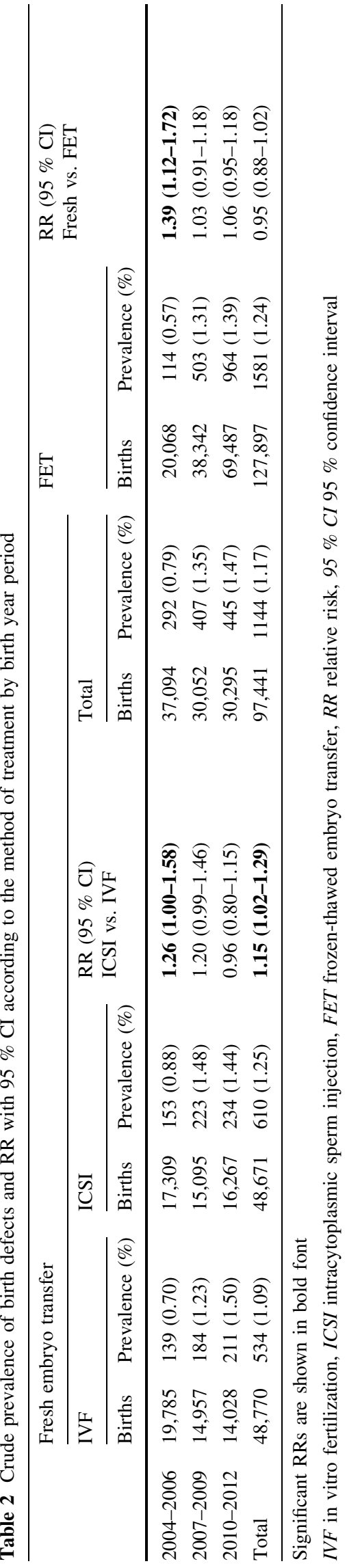




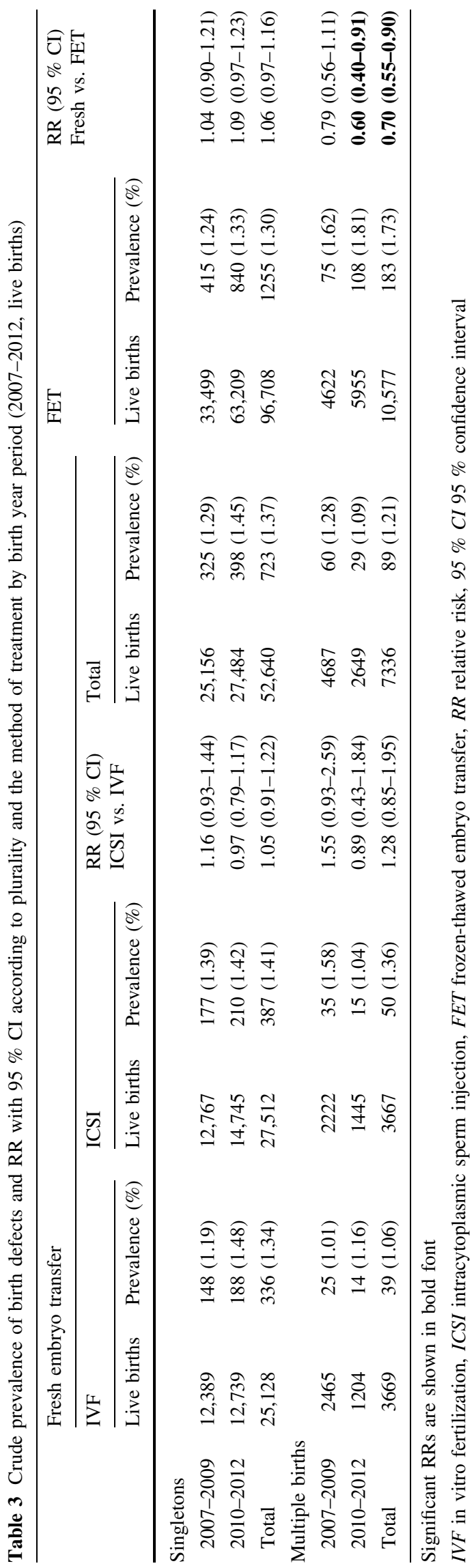

compare the prevalence of birth defect across different time-periods or populations. Therefore, the comparison of birth defects in different treatments is biased only if there is differential reporting according to the method of treatment, which is not likely to occur this type of national data.

The main results of the present study were the following. First, regarding fresh embryo transfer, ICSI might have more risk of birth defects compared with IVF, at least this was true in the earlier period (2004-2006). There are two meta-analyses of whether birth defects are more common in ICSI compared with IVF infants $[8,9]$ and both suggest no significant difference in risk between the two methods. Lie et al. [8] combined the results of four studies to obtain pooled estimates of birth defect risk in ICSI compared with IVF infants of 1.12 (95\% IC 0.97-1.28). According to Wen et al. [9], 24 studies were performed regarding birth defects in children conceived by IVF compared with those by ICSI. Overall no difference in risk for birth defects was found between IVF and ICSI groups $(\mathrm{RR}=1.05,95 \% \mathrm{CI}$ 0.91-1.20). On the other hand, two recent reports $[10,11]$ suggested a higher risk for ICSI compared with IVF. A recent survey in South Australia [10] showed an increased risk for birth defects after ICSI, even adjusting for possible confounding factors, such as year of birth, maternal age, and parity. A Chinese study with a 3-year follow-up [11] showed the same tendency, especially for boys. The risk of IVF vs. ICSI is thus confusing. The present result suggested that the elevated risk of ICSI compared with IVF might exist in some periods in Japan for whatever reason. According to the Pinborg study [12], recent data may show a lower birth defect risk for ICSI because the technique is now used to treat a broader range of infertile couples rather than being restricted to those with severe male infertility.

Second, fresh embryo transfer might also have greater risk than FET in the earlier period (2004-2006). In this point, although the risks of preterm birth, small for gestational age, and low birthweight were all lower for FET compared with fresh transfer in the meta-analysis [13], no significant differences were seen between FET and fresh embryo transfers for birth defects in other meta-analysis [14]. Other single studies [11, 15] also reported no increase in risk of birth defects after FET compared with fresh IVF/ ICSI. On the other hand, a recent Chinese study [11] suggested a lower rate of birth defects in FET children compared with fresh IVF/ICSI. Moreover, a recent study by Davies et al. [10] found regarding ICSI a significant increase in the risk of birth defects associated with fresh embryo cycles but not with frozen-embryo transfer cycles, compared to unassisted conception. These results are often explained by a reduced likelihood that developmentally compromised embryos will survive the thawing process [10] or only the most robust embryos survive the initial 
selection process, leading to superior quality embryos being frozen [11]. When discussing the present results, caution is needed because the total number of FET increased rapidly during the observation period (2004-2012), while that of fresh IVF slightly decreased and that of fresh ICSI remained relatively constant, as shown in Table 2. This difference in observation numbers may provide a simple mathematical explanation for the increase of birth defects in fresh embryo transfer compared with FET.

Third, in the case of multiple births, FET pregnancies, however, have elevated risk compared with fresh embryo transfer, as shown in Table 3. This tendency was not observed in singletons. Although the data were limited to live births in the present study, multiple births in FET may be more vulnerable than singletons. The studies on ART's effects according to the method of treatment and plurality are very limited and suffer from small sample sizes. Of them, Olson et al. [15] reported that cryopreservation of embryos did not seem to have an effect on birth defect rates in singletons as compared with singletons conceived after the transfer of fresh embryos with IVF. There was, however, a higher incidence of birth defects in twins born after transfer of cryopreserved embryos as compared with twins born after the transfer of fresh embryos $(\mathrm{RR}=2.11,95 \%$ CI 1.02-4.33) [15]. On the other hand, Belva et al. [16] reported that only cryo-ICSI singletons as compared with fresh ICSI singletons had significantly higher malformation rates, and this tendency was not observed regarding multiple births. Although the percentage of multiple pregnancy in ART is dramatically decreased because of the widespread adoption of the single-embryo transfer policy [6], the present result suggested that plurality should be considered when analyzing the relationship between the effect of the each ART treatment on birth defects.

This study has the following limitations, most of which could be attributed to the dataset: namely, the fact that individual information was obtained only from subjects with birth defects after ART, not the total ART pregnancies.

The first and greatest limitation is that the author could not control for confounding factors that can affect ART and/or birth defects, such as maternal age, parity, smoking, and socioeconomic status, medical history, and prenatal care, since these data on general ART populations are not available. Therefore, whether the results were attributable to the characteristics of patients, other descriptive epidemiological factors (regarding time, place, and person) and/or ART techniques themselves was unclear. Therefore, present results should be considered as the total effects of ART on birth defects.

Second, the author could not check the reliability of the data directly. Several misspellings or misclassifications of diseases were found in the annual report. This is the essential limitation of secondary data analyses.

Third, follow-up after birth was limited to the early neonatal period, and was incomplete. The prevalence of birth defects increases with the growth of children, since some birth defects are not obvious within a few days after birth.

Even with all these limitations, the present results overviewed some characteristics of births defects after ART according to the type of treatment using nationwide multi-year data in Japan. As mentioned earlier, the percentage of children born after ART is now about $4 \%$ in Japan. This value is nearly the same as the percentage of women who smoke during pregnancy. However, the study of ART's influence on later life, especially for the longterm influence of children, is still limited in Japan. Considering the widespread use of fertility treatment, the method of pregnancy, namely spontaneous conception vs. medically assisted reproduction (MAR), including both ovulation induction and ART (each method of ART treatment, if possible), would become more important factors when analyzing intrauterine environmental, or in some cases genetic/epigenetic factors that influence or modify the later development of children. This would be especially important when verifying fetal origin of adult disease (FOAD) or developmental origin of health and disease (DOHaD) hypotheses. In conclusion, the present study suggested that the impacts of different ART methods on birth defects might differ. Proper registration and long-term follow-up after ART and population-based epidemiological researches are needed.

Acknowledgments I would like to thank Toshimi Ooma for assistance with data analysis. This work was supported by JSPS KAKENHI Grant Number 15H04785.

\section{Compliance with ethical standards}

Conflict of interest The authors declare no conflict of interest. This article does not contain any studies with human participants performed by any of the authors.

\section{References}

1. Mayor S. Risk of congenital malformations in children born after assisted reproduction is higher than previously thought. BMJ. 2010;340:c3191. doi:10.1136/bmj.c3191.

2. http://www.who.int/mediacentre/factsheets/fs370/en/. Accessed 17 July 2015.

3. Rimm AA, Katayama AC, Diaz M, Katayama KP. A metaanalysis of controlled studies comparing major malformation rates in IVF and ICSI infants with naturally conceived children. J Assist Reprod Genet. 2004;21(12):437-43.

4. Hansen M, Bower C, Milne E, de Klerk N, Kurinczuk JJ. Assisted reproductive technologies and the risk of birth defects-a systematic review. Hum Reprod. 2005;20(2):328-38. 
5. Hansen M, Kurinczuk JJ, Milne E, de Klerk N, Bower C. Assisted reproductive technology and birth defects: a systematic review and meta-analysis. Hum Reprod Update. 2013;19(4):330-53. doi:10.1093/humupd/dmt006.

6. Ooki S. Birth defects in singleton versus multiple ART births in Japan (2004-2008). J Pregnancy. 2011;2011:285706. doi:10. $1155 / 2011 / 285706$.

7. Källén B, Finnström $\mathrm{O}$, Lindam A, Nilsson E, Nygren KG, Otterblad PO. Congenital malformations in infants born after in vitro fertilization in Sweden. Birth Defects Res A Clin Mol Teratol. 2010;88(3):137-43. doi:10.1002/bdra.20645.

8. Lie RT, Lyngstadaas A, Ørstavik KH, Bakketeig LS, Jacobsen G, Tanbo T. Birth defects in children conceived by ICSI compared with children conceived by other IVF-methods; a meta-analysis. Int J Epidemiol. 2005;34(3):696-701.

9. Wen J, Jiang J, Ding C, Dai J, Liu Y, Xia Y, et al. Birth defects in children conceived by in vitro fertilization and intracytoplasmic sperm injection: a meta-analysis. Fertil Steril. 2012;97(6):1331-7. doi:10.1016/j.fertnstert.2012.02.053.

10. Davies MJ, Moore VM, Willson KJ, Van Essen P, Priest K, Scott $\mathrm{H}$, et al. Reproductive technologies and the risk of birth defects. N Engl J Med. 2012;366(19):1803-13. doi:10.1056/ NEJMoa1008095.

11. Yin L, Hang F, Gu LJ, Xu B, Ma D, Zhu GJ. Analysis of birth defects among children 3 years after conception through assisted reproductive technology in China. Birth Defects Res A Clin Mol Teratol. 2013;97(11):744-9. doi:10.1002/bdra.23116.
12. Pinborg A, Loft A, Henningsen AK, Ziebe S. Does assisted reproductive treatment increase the risk of birth defects in the offspring? Acta Obstet Gynecol Scand. 2012;91(11):1245-6. doi:10.1111/j.1600-0412.2012.01500.x.

13. Wennerholm UB, Söderström-Anttila V, Bergh C, Aittomäki K, Hazekamp J, Nygren KG, et al. Children born after cryopreservation of embryos or oocytes: a systematic review of outcome data. Hum Reprod. 2009;24(9):2158-72. doi:10.1093/humrep/ dep125.

14. Maheshwari A, Pandey S, Shetty A, Hamilton M, Bhattacharya S. Obstetric and perinatal outcomes in singleton pregnancies resulting from the transfer of frozen thawed versus fresh embryos generated through in vitro fertilization treatment: a systematic review and meta-analysis. Fertil Steril. 2012;98(2):368-77. doi:10.1016/j.fertnstert.2012.05.019.

15. Olson CK, Keppler-Noreuil KM, Romitti PA, Budelier WT, Ryan $\mathrm{G}$, Sparks AE, et al. In vitro fertilization is associated with an increase in major birth defects. Fertil Steril. 2005;84(5):1308-15.

16. Belva F, Henriet S, Van den Abbeel E, Camus M, Devroey P, Van der Elst J, et al. Neonatal outcome of 937 children born after transfer of cryopreserved embryos obtained by ICSI and IVF and comparison with outcome data of fresh ICSI and IVF cycles. Hum Reprod. 2008;23(10):2227-38. doi:10.1093/humrep/ den254. 